\title{
Paralytic rabies mimicking Guillain-Barré syndrome: the dilemma still prevails
}

Rohini M. Surve, MD, PDF ; Hima S. Pendharkar, MD, DM²; Sonia Bansal, MD, DNB, PDF

'Department of Neuroanaesthesia and Neurocritical Care, National Institute of Mental Health and Neurosciences (NIMHANS), Bengaluru, India

${ }^{2}$ Department of Neuro Imaging and Interventional Radiology, National Institute of Mental Health and Neurosciences (NIMHANS), Bengaluru, India

\section{Journal of Neurocritical Care \\ CASE REPORT \\ Received: January 28, 2021 \\ Revised: March 5, 2021 \\ Accepted: March 13, 2021 \\ Corresponding Author: Rohini M. Surve, MD, PDF \\ Department of Neuroanaesthesia and Neurocritical Care, National Institute of Mental Health and Neurosciences (NIMHANS), Neurocentre Faculty Block, 3rd Floor, Hosur Rd, Bengaluru 560029, India \\ Tel: +91-80-2699-5415 \\ Fax: +91-80-2656-4830 \\ E-mail: rohinigondhule@gmail.com}

Background: Paralytic rabies lacks the hallmark signs of rabies at presentation and is often misdiagnosed as Guillain-Barre Syndrome (GBS). Sensitive antemortem diagnostic criteria for rabies is lacking, and the diagnosis is confirmed posthumously by demonstrating Negri bodies on brain biopsy. Antemortem brain and spine magnetic resonance imaging findings have recently been reported for paralytic rabies.

Case Report: We report a case of paralytic rabies in a young boy who was initially misdiagnosed with GBS. In this case, brain and spine magnetic resonance imaging (MRI) findings pointed towards paralytic rabies, which was confirmed by the presence of anti-rabies antibodies in the serum and cerebrospinal fluid analysis, and posthumously by Negri bodies on brain biopsy.

Conclusion: In patients with GBS, paralytic rabies should be considered as an alternative diagnosis, especially in regions where rabies is endemic, and early MRI of the brain and spine should be considered for the antemortem diagnosis of paralytic rabies.

Keywords: Paralytic rabies; Guillain-Barré syndrome; Magnetic resonance imaging

\section{INTRODUCTION}

Rabies leads to nearly 59,000 human deaths annually in over 150 countries [1]. Despite rabies being a fulminant problem with a high fatality rate, diagnosis of rabies, especially atypical or paralytic rabies, remains a challenge. This could be due to lack of hallmark signs of aerophobia/hydrophobia, autonomic disturbances, and initial presentation as acute flaccid paralysis (AFP) mimicking Guillain-Barré Syndrome (GBS) [2]. Further, lack of exposure history (cryptogen- ic) adds to the diagnostic problem [2]. We present a case of cryptogenic paralytic rabies mimicking GBS, thus delaying the diagnosis.

\section{CASE REPORT}

An 18-year-old boy presented with a history of fever for the past 7 days, weakness in the lower limbs for the last 5 days, and weakness in the upper limbs and difficulty in swallowing for the past 3 days. The patient was drowsy, but followed commands. He was unable

(C) 2021 The Korean Neurocritical Care Society

This is an Open Access article distributed under the terms of the Creative Commons Attribution Non-Commercial License (http://creativecommons.org/licenses/by-nc/4.0/) which permits unrestricted noncommercial use, distribution, and reproduction in any medium, provided the original work is properly cited. 
to lift his head and had a poor cough reflex and shallow respiration. The single breath count (an estimate of vital capacity; normal range, 25-30) was 5. Neck rigidity was not observed. Patient was areflexic, with mute plantar responses. The Medical Research Council grade for motor power was $2 / 5$ and $0 / 5$ for upper limbs and lower limbs, respectively, with normal sensory examination. Pupils were equal, reactive to light, and the fundus was normal upon examination. Laboratory investigations were normal except for serum sodium, which was $121 \mathrm{mmol} / \mathrm{L}$ (normal, 135-147 $\mathrm{mmol} / \mathrm{L}$ ). There was no history of dog bite, bat bite, or recent vaccination. A preliminary diagnosis of GBS was made, and large-volume plasmapheresis (LVPP) was planned.

Drowsiness was attributed to hyponatremia, and correction with $3 \% \mathrm{NaCl}$ was initiated. Although the patient's sensorium improved by the next day, he became quadriplegic. The patient underwent tracheal intubation, and mechanical ventilation was initiated. The patient received four cycles of LVPP on alternate days. Cerebrospinal fluid (CSF) analysis revealed six cells; protein, 26 $\mathrm{mg} / \mathrm{dL}$; glucose, $82 \mathrm{mg} / \mathrm{dL}$; and chloride, $113 \mathrm{mmol} / \mathrm{L}$ (normal CSF contains 0-5 white blood cells, $15-45 \mathrm{mg} / \mathrm{dL}$ protein, 40 $70 \mathrm{mg} / \mathrm{dL}$ glucose, $118-132 \mathrm{mmol} / \mathrm{L}$ chloride). The chronic meningitis workup was also normal. Nerve conduction studies were planned but could not be performed due to logistic reasons. On the sixth day, the patient became drowsy and developed hypothermia and hypotension, requiring external forced air warming and noradrenaline infusion. Computed tomography of the brain revealed normal findings. Furthermore, extraocular movements became restricted in all directions, and the patient developed vertical nystagmus and pupillary asymmetry with sluggish reaction to light. In view of these signs, diagnosis was reconsidered to be either acute demyelinating encephalomyelitis (ADEM) or viral encephalitis; therefore, intravenous methylprednisolone 30 $\mathrm{mg} / \mathrm{kg} /$ day and acyclovir were started on day 9. Simultaneously, magnetic resonance imaging (MRI) of the brain and spine was performed, and serum samples were sent for antibody testing to investigate infectious causes, such as Herpes simplex virus encephalitis, Japanese encephalitis, chikungunya, syphilis, hepatitis $\mathrm{B}$, dengue, or rabies.

MRI of the brain (T2 weighted and fluid-attenuated inversion recovery) and spine (plain and contrast) revealed the involvement of cerebral cortical gray matter, midbrain, optic chiasma, hypothalamic region (Fig. 1), and central gray matter of the spinal cord in the lower dorsal and lumbar region with intense post-contrast enhancement of nerve roots of the cauda (Fig. 2), respectively. MRI findings were suggestive of paralytic rabies. Subsequently, the patient became deeply comatose, with pupils dilated, fixed, and unreactive to light. The patient died on day 15 . Salivary polymerase chain reaction (PCR) for rabies viral RNA was negative on day 12. However, the rabies antibodies test by rapid fluorescent focus inhibition technique (RFFIT) came out positive on day 13 with a serum titer of 4,096 IU/mL (normally titers are not detectable if the patient is not vaccinated for rabies). The patient's father later confirmed a history of dog bite 2 years back on his left leg in the school (as told by his friends). The patient had not informed about this at home and was not vaccinated for rabies. The patient's parents refused an autopsy but agreed for transnasal brain biopsy and lumbar puncture. Post-mortem CSF-PCR for rabies virus was negative. However, the CSF-RFFIT titer for antibodies was $<16$ $\mathrm{IU} / \mathrm{mL}$ (detectable titers in the CSF is considered as positive for rabies irrespective of the vaccination status), and brain biopsy showed Negri bodies, confirming the diagnosis of rabies.

\section{DISCUSSION}

Our patient initially presented with rapidly progressing AFP, mimicking GBS, without any preceding history of animal bite (positive history surfaced just a day prior to his death). Although GBS is the most common cause of AFP, it is important to consider rabies as a differential diagnosis at the earliest, even in the absence of a history of animal bite, especially in regions where rabies is endemic $[1,2]$. In one of the largest retrospective studies, involving 34 patients with paralytic rabies over 30 years, authors reported that the most common misdiagnosis was GBS (17/34) followed by ADEM (10/34) based on the patients' initial clinical presentation [2]. There are also a few similar case reports [3-5]. Gadre et al. [2] observed that the incubation period between the time of animal bite and onset of rabies symptoms ranged from 7 days to 4 years. Paralytic rabies frequently presents as GBS, leading to a diagnostic dilemma. Furthermore, due to the lack of antemortem diagnostic criteria for rabies, the diagnosis remains challenging. Clinically, the presence of progressive paralysis with highgrade fever, bladder involvement, paraesthesia, rapid progression to cranial nerve palsies, altered mental status, deep coma, and the absence of albumin-cytological dissociation in CSF on laboratory examination, should raise the suspicion of paralytic rabies [2]. Major difference in the clinical course of paralytic rabies and furious rabies is attributed to the host immune response to the virus, which is defective in paralytic rabies and heightened in a furious form [6]. If we treat paralytic rabies with plasma therapy or immunoglobulins on the pretext of GBS, the possibility of protracted clinical course of the paralytic rabies and delayed encephalitic manifestations cannot be ruled out. It has been observed that immunotherapy and post-exposure prophylaxis can sometimes delay rabies manifestations and can present as paralytic rabies mim- 

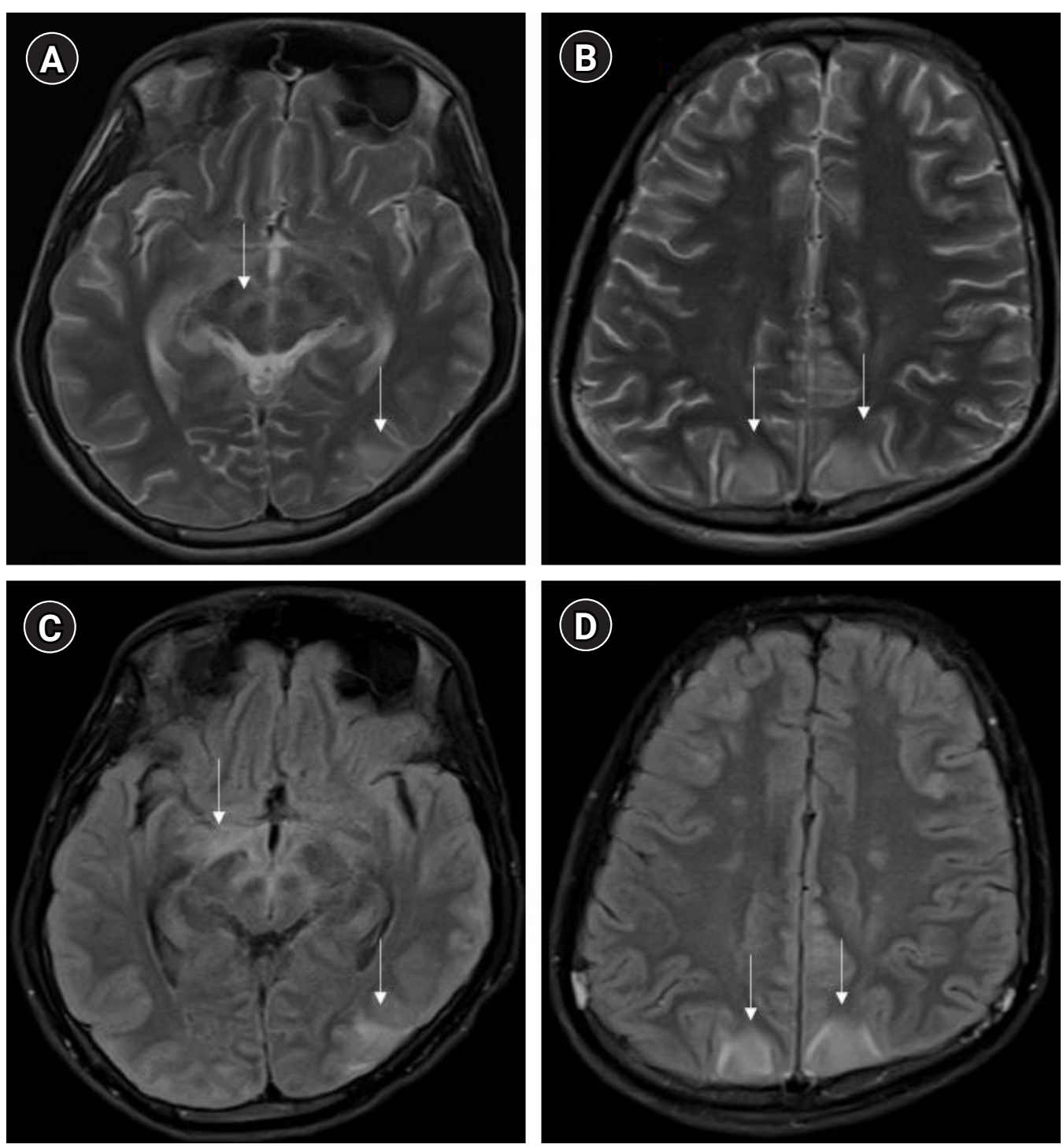

Fig. 1. Magnetic resonance imaging brain-T2 weighted axial image at the level of midbrain shows hyperintensity along the substantia nigra, mesial temporal structures, and left parieto-occipital region involving cortex and underlying white matter (A), at a higher level shows bilateral parieto-occipital hyperintensities involving the cortex and underlying white matter; subtle hyperintensities are also noted in centrum semiovale bilaterally (B). On fluid-attenuated inversion recovery axial images, the hyperintensities are better appreciated in the similar regions and additional hyperintensities are noted in the optic tracts and hypothalamic region (C) and bilateral frontal region (not apparent on T2) (D). Pathological changes are highlighted by vertical arrows.

icking GBS [7].

Antemortem diagnosis of rabies requires detection of viral RNA (in CSF, saliva, or skin) or neutralizing antibodies (in CSF or serum) by various methods, which are specific but have their own limitations with respect to sensitivity, turn-around time, availability, and affordability [8]. MRI findings in rabies have been found to be promising for diagnosing and distinguishing it from other common forms of AFP. As per available literature, MRI of paralytic rabies shows its predilection for specific areas, such as spinal cord, thalamus, hypothalamus, basal ganglia, brainstem, and hippocampus, which shows hyperintensity on T2 and flu- id-attenuated inversion recovery [2-4]. In GBS, imaging findings are limited to nerve root enhancement in the cauda equina, sparing the brain parenchyma [9]. Imaging in ADEM predominantly involves white matter, although there can be cortical and deep gray matter involvement [10]. On the other hand, the hallmark of rabies is cerebral cortical and spinal gray matter involvement with relative sparing of the white matter, which may help differentiate rabies encephalitis from demyelinating disorders. Rabies encephalitis and paralytic rabies have similar MRI findings, although spinal cord and medullary involvement is more commonly observed in the paralytic form [11]. In our case, we found involvement of 

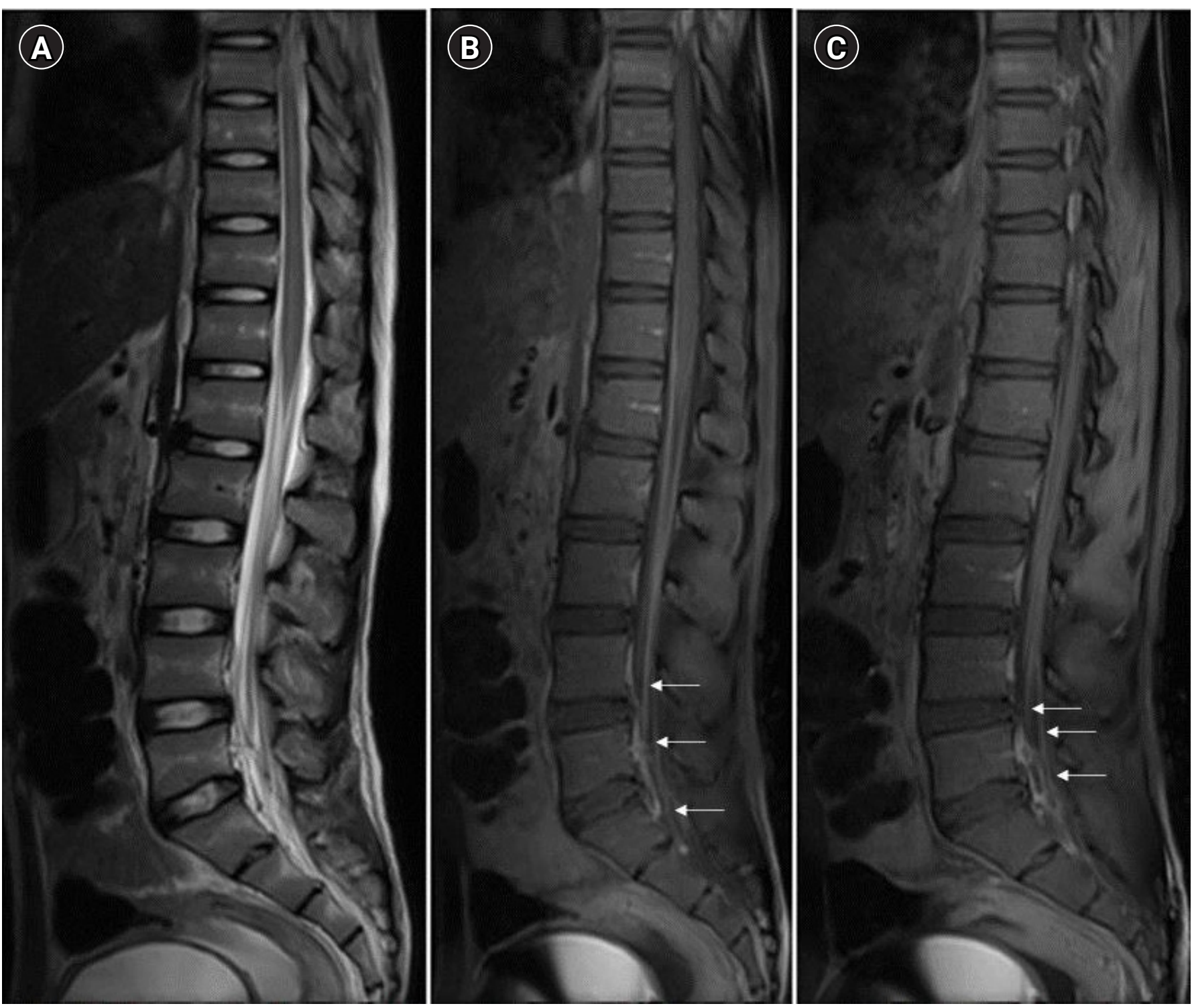

Fig. 2. Magnetic resonance imaging spine-T2 weighted sagittal image of the conus-cauda region shows subtle hyperintense signal changes in the conus and nerve roots of the cauda are slightly thickened (A). Post-contrast T1 fat saturation image shows intense enhancement of the cauda nerve roots along with conus enhancement (B) and nerve roots enhancement noted at parasagittal level (C), as indicated by horizontal arrows.

the mid brain, optic chiasma, hypothalamic region, and gray matter of the spinal cord with nerve root enhancement. Our MRI findings resonate with the existing literature on MRI in paralytic rabies.

Early diagnosis of paralytic rabies is crucial to prevent unnecessary treatment on the lines of GBS or other demyelinating disorders and to explain the prognosis to the family. This will enable us to take appropriate infection control measures. In addition, with the emerging cases of rabies survivors, early diagnosis can give an impetus in trying newer treatment modalities in addition to supportive care $[11,12]$. To prevent neuronal damage following viral infiltration and subsequent immune response, various treatment protocols have been tried in the past, which include induction of therapeutic coma or hypothermia, but they were not found to be effective [13]. Sedatives, such as barbiturates, ketamine, or midazolam, and other medications, such as amantadine, antiviral (ribavirin), high dose immunoglobulins, and interferon alpha, also did not show promising results $[13,14]$. Newer therapeutic approaches include the use of newer antivirals such as favipiravir, molecular engineering of rabies antibodies for passive immunity, exogenous cytokines, and more recently silencing RNA and artificial microRNAs are being investigated and need further exploration to test the possible protocol that will work in rabies management [13].

In a patient diagnosed with GBS, paralytic rabies should be 
considered as a potential differential diagnosis, even in the absence of a history of animal bite, especially if the patient belongs to a rabies endemic region. To facilitate early antemortem diagnosis of rabies, MRI of the brain and spine should be considered along with the antemortem rabies-specific tests.

\section{ARTICLE INFORMATION}

\section{Ethics statement}

This case was reviewed and approved by the Institute Ethics Committee (IEC) of National Institute of Mental Health and Neurosciences (No. NIMHANS/IEC/2020-21 dated 02.12.2021). Written informed consent was obtained from the patient's father.

\section{Conflict of interest}

No potential conflict of interest relevant to this article.

\section{ORCID}

Rohini M. Surve https://orcid.org/0000-0002-5740-7122

Hima S. Pendharkar https://orcid.org/0000-0002-8711-7708

Sonia Bansal https://orcid.org/0000-0003-2872-8629

\section{Author contributions}

Conceptualization: RMS. Data curation: RMS. Formal analysis: RMS, HSP. Writing-original draft: RMS. Writing-review \& editing: all authors.

\section{Additional contributions}

We thank the deceased patient's father for providing consent to publish this case report.

\section{REFERENCES}

1. World Health Organization. Rabies: epidemiology and burden of disease [Internet]. Geneva: World Health Organization; 2010 [cited 2021 Mar 31]. Available from: https://www.who. int/rabies/epidemiology/en/.

2. Gadre G, Satishchandra P, Mahadevan A, Suja MS, Madhusudana SN, Sundaram C, et al. Rabies viral encephalitis: clinical determinants in diagnosis with special reference to paralytic form.J Neurol Neurosurg Psychiatry 2010;81:812-20.

3. Kumar N, Gupta P, Meena MK. Paralytic rabies: a Guillain-Barre syndrome mimic. QJM 2019;112:365-6.

4. Kalita J, Bhoi SK, Bastia JK, Lashkar S, Mahadevan A, Misra UK. Paralytic rabies: MRI findings and review of literature. Neurol India 2014;62:662-4.

5. Sheikh KA, Ramos-Alvarez M, Jackson AC, Li CY, Asbury AK, Griffin JW. Overlap of pathology in paralytic rabies and axonal Guillain-Barre syndrome. Ann Neurol 2005;57:768-72.

6. Hemachudha T, Panpanich T, Phanuphak P, Manatsathit S, Wilde H. Immune activation in human rabies. Trans R Soc Trop Med Hyg 1993;87:106-8.

7. Mahadevan A, Suja MS, Mani RS, Shankar SK. Perspectives in diagnosis and treatment of rabies viral encephalitis: insights from pathogenesis. Neurotherapeutics 2016;13:477-92.

8. Mani RS, Madhusudana SN. Laboratory diagnosis of human rabies: recent advances. ScientificWorldJournal 2013;2013: 569712.

9. Alkan O, Yildirim T, Tokmak N, Tan M. Spinal MRI findings of Guillain-Barre syndrome.J Radiol Case Rep 2009;3:25-8.

10. Marin SE, Callen DJ. The magnetic resonance imaging appearance of monophasic acute disseminated encephalomyelitis: an update post application of the 2007 consensus criteria. Neuroimaging Clin N Am 2013;23:245-66.

11. Rao A, Pimpalwar Y, Mukherjee A, Yadu N. Serial brain MRI findings in a rare survivor of rabies encephalitis. Indian J Radiol Imaging 2017;27:286-9.

12. Netravathi M, Udani V, Mani RS, Gadad V, Ashwini MA, Bhat $\mathrm{M}$, et al. Unique clinical and imaging findings in a first ever documented PCR positive rabies survival patient: a case report. J Clin Virol 2015;70:83-8.

13. El-Sayed A. Advances in rabies prophylaxis and treatment with emphasis on immunoresponse mechanisms. Int J Vet Sci Med 2018;6:8-15.

14. Hemachudha T, Ugolini G, Wacharapluesadee S, Sungkarat W, Shuangshoti S, Laothamatas J. Human rabies: neuropathogenesis, diagnosis, and management. Lancet Neurol 2013;12:498513. 